$>$ Nous poursuivons dans ce numéro la publication des Nouvelles rédigées par les étudiants du Master «Biologie Santé », dans le cadre du module "physiopathologie de la signalisation » de l'université Paris-Saclay. Ces textes s'inscrivent dans le développement d'un partenariat que médecine/sciences propose aux Écoles doctorales (voir à ce propos l'éditorial de Hervé Chneiweiss, $\mathrm{m} / \mathrm{s} \mathrm{n}^{\circ}$ 6-7, juin-juillet 2016 , page 537). Après les quatre Nouvelles publiées dans le numéro de juin-juillet 2016, précédées d'une introduction des enseignants participants, voici une seconde série de cinq Nouvelles. Une troisième série sera publiée en octobre 2016 . Toutes ces Nouvelles sont en accès libre sur le site de $\mathrm{m} / \mathrm{s}$ (www.medecine/ sciences.org). Bonne lecture! <
Partenariat médecine/sciences Écoles doctorales (2)

L'actualité scientifique vue par les étudiants du Master Biologie Santé, module physiopathologie de la signalisation, Université Paris-Saclay

\begin{tabular}{|c|c|c|}
\hline UnIVersIte $_{\text {PARIS-SACLAY }}$ & $\begin{array}{l}\text { SCHOOL } \\
\text { BIOLOGIE, MÉDECINE, } \\
\text { PHARMACIE }\end{array}$ & $\begin{array}{l}\text { MASTER } \\
\text { Biologie Santé }\end{array}$ \\
\hline
\end{tabular}

Karim Benihoud (professeur, université Paris-Sud) Sophie Dupré (maître de conférences, université Paris-Sud) Olivier Guittet (maître de conférences, université Paris-Sud) Boris Julien (maître de conférences, université Paris-Sud) Hervé Le Stunff (professeur, université Paris-Sud) Philippe Robin (maître de conférences, université Paris-Sud) karim.benihoud@u-psud.fr

Série coordonnée par Laure Coulombel.

\title{
NOUVELLE
}

\section{Lien entre la protéine suppresseur de tumeur p53 et la biogenèse des centres Fe-S}

Alexis Andrél ${ }^{\star}$, Marion Antonini ${ }^{\star *}$, Marie-Pierre Golinelli-Cohen ${ }^{2}$
${ }^{1}$ Ml Biologie Santé, Université Paris-Saclay, 91405 Orsay, France ;

${ }^{2}$ Institut de chimie des substances naturelles, CNRS UPR2301, université Paris-Sud, université Paris-Saclay, 91190 Gif-surYvette, France.

* Ces deux auteurs ont participé également à la rédaction de cette Nouvelle.

alexis.andre@u-psud.fr

marionantonini@gmail.com

marie-pierre.golinelli@cnrs.fr
Aujourd'hui, près de 400000 nouveaux cancers sont diagnostiqués chaque année en France. Cependant, on ne guérit actuellement qu'un tiers des patients, alors qu'un des objectifs est d'en guérir le double d'ici 2025. L'identification de nouvelles cibles thérapeutiques est par conséquent absolument fondamentale.

Cette Nouvelle fait partie d'une série de 15 Nouvelles rédigées par les étudiants du Master «Biologie Santé » de l'université Paris-Saclay qui paraîtront dans les numéros $6-7,8-9$ et 10 (2016) de médecine/sciences.
Dans une cellule normale, la protéine suppresseur de tumeur p53 est stabilisée en réponse à différents stress et peut exercer sa fonction de facteur de transcription régulant des fonctions cellulaires importantes comme la mort cellulaire programmée (apoptose) [1]. Cependant, dans près de $50 \%$ des cancers, on trouve une mutation du gène P53. Afin de découvrir de nouvelles cibles de p53, Funauchi et al. [2] ont entrepris une étude par puce à ADN qui a permis l'identification de 60 gènes, dont ISCU (iron-sulfur cluster assembly enzyme), un gène impliqué dans la biogenèse des centres $\mathrm{Fe}-\mathrm{S}$ $[3](\rightarrow)$.

Homéostasie du fer et genèse des centres Fe-S

Le fer est un élément essentiel et une carence conduit au dysfonctionnement de nombreux processus vitaux comme la respiration, le transport de l'oxygène et la réparation de I'ADN, processus dépendants de protéines ayant un cofacteur contenant du fer (hèmes, 


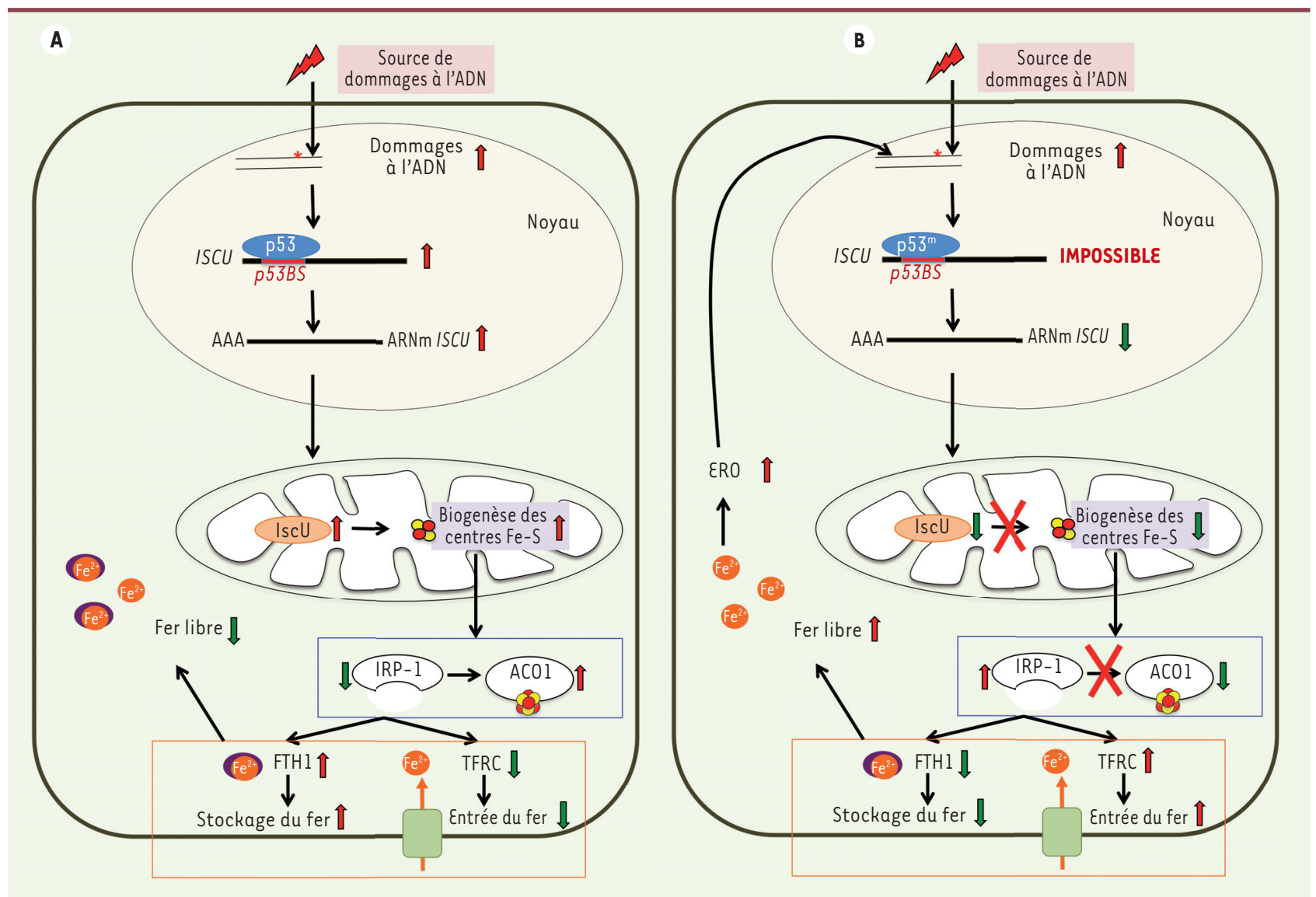

Figure 1. Régulation de la concentration en fer libre intracellulaire par p53. A. Dans une cellule normale, p53 s'accumule lors de dommages à l'ADN, et active notamment la transcription de ISCU. La protéine IscU stimule alors la biogenèse des centres Fe-S avec comme conséquence la conversion d'IRP-1 en ACOl, ce qui modifie la régulation des protéines cibles de l'IRP-1, lève l'inhibition de la transcription de FTHI et déstabilise les transcrits TFRC ; la concentration en fer libre intracellulaire diminue. B. Si p53 mutée ne peut pas se fixer sur ISCU, alors la biogenèse des centres Fe-S n'est plus stimulée et la concentration en IRP-1 ne diminue pas. Le taux des protéines cibles est modifié à l'inverse de précédemment. Le stockage diminue, le fer libre s'accumule et induit la formation des $\varepsilon R 0$ qui vont augmenter les dommages à l'ADN. FTHI : ferritin, heavy polypeptide 1 ; TFRC : transferrin receptor; IRP-1 : iron regulatory protein-1; ACOI : aconitase cytosolique ; IscU : iron-sulfur cluster assembly enzyme ; ERO : espèces réactives de l'oxygène.

centres Fe-S, etc.). Cependant, le fer libre est aussi à l'origine de la formation d'espèces réactives de l'oxygène (ERO), tel le radical libre $\mathrm{OH}^{\bullet}$, qui induisent notamment des dommages à l'ADN [4].

C'est pourquoi l'homéostasie du fer est finement régulée par plusieurs mécanismes. L'un d'entre eux fait intervenir une protéine bifonctionnelle, l'aconitase cytosolique (ACOI)/IRP-1 (iron regulatory protein-1), qui bascule de la forme contenant un centre $\mathrm{Fe}-\mathrm{S}$ (ACOl) à une forme sans centre Fe-S (IRP-1) qui est un régulateur post-transcriptionnel de protéines impliquées dans l'homéostasie du fer. Ainsi, IRP-1 se fixe, soit en 5' d'un
ARN messager (ARNm) dont il inhibe l'expression (cas de la ferritine, FTHl [FTHI : ferritin, heavy polypeptide 1]), impliquée dans le stockage du fer, soit en 3' d'un ARNm qu'il stabilise (cas du récepteur de la transferrine, TFRC [TFRC : transferrin receptor], impliqué dans l'import du fer) [5]. Il est désormais admis que l'accumulation de fer dans les cellules augmente le risque de certains cancers hépatiques et colorectaux, et de nouvelles stratégies thérapeutiques utilisant des chélateurs de fer commencent à apparaître [6]. Or, ISCU, un des gènes cibles de p53 identifié par cette étude, intervient dans la biogenèse des centres Fe-S.
Un lien entre p53 et la genèse des centres Fe-S

Funauchi et al. [2] ont ensuite révélé le lien entre p53 et la genèse des centres Fe-S. Ainsi, la surexpression de p53, ou son activation par induction de dommages à l'ADN, augmentent les quantités de transcrits ISCU et de la protéine correspondante. 0r, p53 induit l'expression de ses gènes cibles en se liant à un motif ( $p 53 \mathrm{BS}$ ) très conservé situé sur ces même gènes. Un tel motif a été identifié dans le premier intron de ISCU. p53BS a alors été introduit dans une séquence promotrice en amont d'un gène rapporteur codant pour la luciférase. Ainsi, l'augmentation 
de l'expression de la luciférase en présence de p53 a confirmé que p53BS est bien un site de liaison à $p 53$, interaction qui peut être détruite, notamment par introduction d'une mutation dans p53, mutation couramment rencontrée dans les cancers. Ainsi, IscU est clairement une nouvelle cible de la protéine antiapoptotique $\mathrm{p} 53$.

L'insertion d'un centre $\mathrm{Fe}-\mathrm{S}$ dans ACOl/ IRP-1, et donc l'équilibre entre les deux formes avec ou sans centre $\mathrm{Fe}-\mathrm{S}$, sont liés aux machineries de biogenèse des centres Fe-S, dont IscU fait partie [7]. L'effet du niveau protéique de IscU sur deux protéines régulées par IRP-1, FTHI et TFRC, a alors été étudié. Ainsi, l'extinction de l'expression de IscU par ARN interférence dans des cellules en culture s'accompagne d'une baisse de FTHI via une augmentation de la fixation d'IRPl sur les ARNm de FTHI et de TFRC. À l'inverse, l'activation de p53 - qui aboutit à une augmentation des transcrits et de la protéine IscU - conduit à une baisse de TFRC et à une augmentation de FTHl. Ces données sont validées dans des tissus primaires : dans des cellules cancéreuses déplétées en $p 53$, une augmentation significative du fer intracellulaire (baisse du stockage, augmentation de l'entrée intracellulaire) est observée. II en est de même pour le fer plasmatique chez des souris déplétées en p53 et soumises à un régime riche en fer. Pour finir, plus de la moitié des tissus cancéreux hépatiques étudiés présentent une baisse significative du niveau de IscU, observable uniquement si p53 est mutée.
La régulation de la biogenèse des protéines Fe-S par la protéine suppresseur de tumeur $\mathrm{p} 53$ : un maillon essentiel du maintien de l'intégrité génomique Des études antérieures avaient montré le lien entre $p 53$ et la régulation du fer par IRP-1 [8], mais le mécanisme exact n'était pas connu. Cette nouvelle étude révèle un mécanisme en montrant clairement que IscU, protéine de la machinerie de biogenèse des protéines $\mathrm{Fe}-\mathrm{S}$, est une cible de p53. Ainsi, l'activation de p53 augmente l'expression de IscU qui stimule la biogenèse des centres Fe-S dont la conversion d'IRP-1 en ACOl. La concentration d'IRP-l diminuant, l'expression de TFRC est alors diminuée (diminution de l'entrée du fer), tandis que celle de FTHl est augmentée (augmentation du stockage), ce qui diminue la concentration en fer libre cellulaire (Figure $1 A$ ). Inversement, si p53 mutée ne peut pas se lier au gène ISCU, l'expression de ce dernier n'est alors pas stimulée, le fer libre celIulaire s'accumule, tandis que les ERO et les dommages à I'ADN augmentent (Figure 1B).

Précédemment, il a été montré que la frataxine, autre protéine impliquée dans la biogenèse des centres Fe-S, est, elle aussi, régulée par p53 [9]. Des souris qui n'expriment pas la frataxine développent des tumeurs et présentent une altération des protéines $\mathrm{Fe}-\mathrm{S}$ de réparation de I'ADN [10]. Ainsi, en réduisant les dommages à l'ADN par les ERO tout en stimulant la réparation de I'ADN, la régulation de la biogenèse des protéines
Fe-S par la protéine suppresseur de tumeur p53 apparaît comme un nouveau mécanisme fondamental pour le maintien de l'intégrité de l'information génétique lors de la carcinogenèse. $\diamond$

Crosstallk between the tumor suppressor p53 and Fe-S cluster biogenesis

\section{LIENS D'INTÉRÊT}

Les auteurs déclarent n'avoir aucun lien d'intérêt concernant les données publiées dans cet article.

\section{RÉFÉRENCES}

1. Levine AJ. p53, the cellular gatekeeper for growth and division. Cell $1997 ; 88: 323-31$.

2. Funauchi $y$, Tanikawa C, $y_{i}$ Lo PH, et al. Regulation of iron homeostasis by the p53-ISCU pathway. Sci Rep $2015 ; 5: 16497$

3. Py B, Barras F. Du fer et du soufre dans les protéines. Comment la cellule construit-elle les cofacteurs fer-soufre essentiels à son fonctionnement? Med Sci (Paris) 2014 ; $30: 1110-22$.

4. Keyer K,Imlay JA. Superoxide accelerates DNA damage by elevating free-iron levels. Proc Natl Acad Sci USA $1996 ; 93: 13635-40$.

5. Muhlenhoff U, Hoffmann B, Richter N, et al. Compartmentalization of iron between mitochondria and the cytosol and its regulation. EurJ Cell Biol 2015 ; $94: 292-308$.

6. Torti SV, Torti FM. Iron and cancer: more ore to be mined. Nat Rev Cancer $2013 ; 13: 342-55$.

7. Lill R, Dutkiewicz R, Freibert SA, et al. The role of mitochondria and the CIA machinery in the maturation of cytosolic and nuclear iron-sulfur proteins. Eur J Cell Biol 2015; 94 : 280-91

8. Zhang F, Wang W, Tsuji Y, et al. Post-transcriptional modulation of iron homeostasis during $\mathrm{p} 53$-dependent growth arrest. J Biol Chem 2008 ; 283 : 33911-8.

9. Shimizu R, Lan NN, Tai $\Pi$, et al. p53 directly regulates the transcription of the human frataxin gene and its lack of regulation in tumor cells decreases the utilization of mitochondrial iron. Gene 2014 ; 551 : 79-85.

10. Thierbach R, Drewes G, Fusser M, et al. The Friedreich's ataxia protein frataxin modulates DNA base excision repair in prokaryotes and mammals. Biochem J 2010 ; $432: 165-72$.

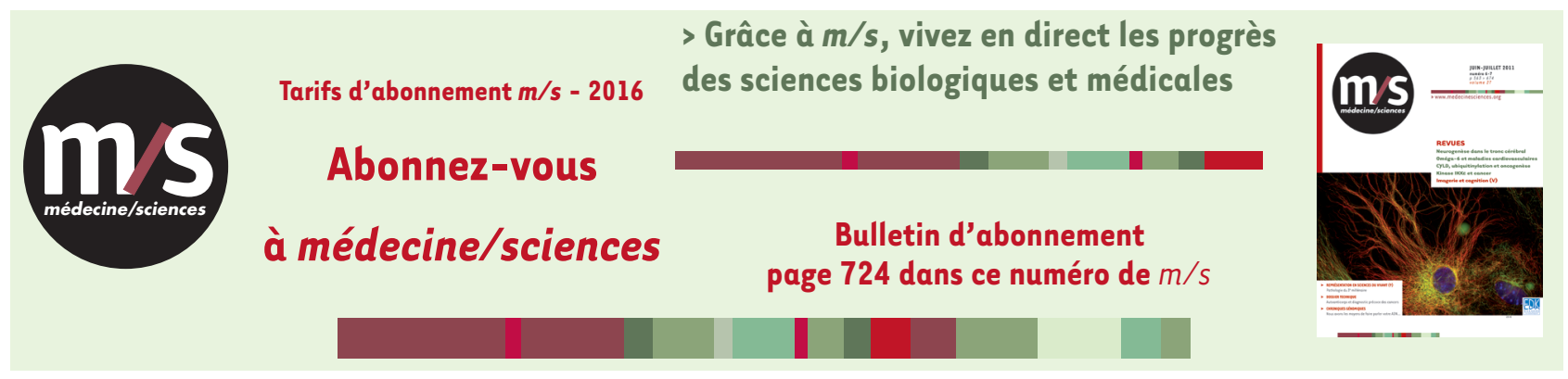

